\title{
UCRL-CONF-217385
}

LA W RENCE LIVERM ORE NATIONAL LABORATORY

\section{Chemistry of $\mathrm{H} 2 \mathrm{O}$ and HF Under Extreme Conditions}

L. Fried, N. Goldman, I-F. W. Kuo, C. Mundy

November 30, 2005

APS Shock Compression of Condensed Matter Baltimore, MD, United States July 31, 2005 through August 5, 2005 
This document was prepared as an account of work sponsored by an agency of the United States Government. Neither the United States Government nor the University of California nor any of their employees, makes any warranty, express or implied, or assumes any legal liability or responsibility for the accuracy, completeness, or usefulness of any information, apparatus, product, or process disclosed, or represents that its use would not infringe privately owned rights. Reference herein to any specific commercial product, process, or service by trade name, trademark, manufacturer, or otherwise, does not necessarily constitute or imply its endorsement, recommendation, or favoring by the United States Government or the University of California. The views and opinions of authors expressed herein do not necessarily state or reflect those of the United States Government or the University of California, and shall not be used for advertising or product endorsement purposes. 


\title{
Chemistry of $\mathrm{H}_{2} \mathrm{O}$ and HF Under Extreme Conditions
}

\author{
Laurence E. Fried, Nir Goldman, I-Feng W. Kuo, Christopher J. Mundy
}

\author{
Lawrence Livermore National Laboratory, P. O. Box 808, Livermore, California 94550
}

\begin{abstract}
The predicted high pressure superionic phases of water and HF are investigated via ab initio molecular dynamics. These phases could potentially be achieved through either static compression with heating or through shock compression. We study water at densities of 2.0-3.0 g/cc (34-115 GPa) along the $2000 \mathrm{~K}$ isotherm. We find that extremely rapid (superionic) diffusion of protons occurs in a fluid phase at pressures between 34 and $58 \mathrm{GPa}$. A transition to a stable body-centered cubic (bcc) O lattice with superionic proton conductivity is observed between 70 and $75 \mathrm{GPa}$, a much higher pressure than suggested in prior work. We find that all molecular species at pressures greater than $75 \mathrm{GPa}$ are too short lived to be classified as bound states. Up to $95 \mathrm{GPa}$, we find a solid superionic phase characterized by covalent $\mathrm{O}-\mathrm{H}$ bonding. Above $95 \mathrm{GPa}$, a transient network phase is found characterized by symmetric O-H hydrogen bonding with nearly $50 \%$ covalent character. Ab initio molecular dynamics simulations of HF were conducted at densities of $1.8-4.0 \mathrm{~g} / \mathrm{cc}$ along the $900 \mathrm{~K}$ isotherm. According to our simulations, a unique form of (symmetric) hydrogen bonding could play a significant role in superionic conduction. Our work shows that superionic phases could be more prevalent in hydrogen bonded systems than previously thought, such as $\mathrm{HCl}$ and $\mathrm{HBr}$.

Keywords: $\mathrm{H}_{2} \mathrm{O}, \mathrm{HF}$, First Principles Molecular Dynamics, Superionic Phase

PACS: $31.15 \mathrm{Ar}, 61.20 \mathrm{Ja}, 71.15 \mathrm{Pd}, 62.50+\mathrm{p}$
\end{abstract}

\section{INTRODUCTION}

Superionic solids are compounds that exhibit exceptionally high ionic conductivity, where one ion type diffuses through a crystalline lattice of the remaining types. This is a unique phase of matter in which chemical bonds are breaking and reforming very rapidly. Since their discovery in 1836 , a fundamental understanding of superionic conductors has been one of the major challenges in condensed matter physics[1]. In general, it has been difficult to create a simple set of rules governing superionic phases. Studies have mostly been limited to metal based compounds, such as metal halides like $\mathrm{AgI}$ and $\mathrm{PbF}_{2}[1]$. However, the existence of superionic solid phases of hydrogen bonded compounds had been theorized previously[2, 3]. Recent experimental and computational results indicate the presence of a high pressure triple point in the $\mathrm{H}_{2} \mathrm{O}$ phase diagram $[4,5,6]$, including a so-called superionic solid phase with fast hydrogen diffusion [6, 7]. Goldman et al. have recently described the emergence of symmetric hydrogen bonding in superionic water at $2000 \mathrm{~K}$ [7] and $95 \mathrm{GPa}$. In symmetric hydrogen bonding, the intramolecular $\mathrm{X}-\mathrm{H}$ bond becomes identical to the intermolecular $\mathrm{X}-\mathrm{H}$ bond, where $\mathrm{X}$ is an electronegative element. It has been suggested that for superionic solids a mixed ionic/covalent bonding character stabilizes the mobile ion during the diffusion process[1]. Symmetric hydrogen bonding provides mixed ionic/covalent bonding, and thus could be a key factor in superionic diffusion in hydrogen bonding systems. This represents an entirely novel approach for creating a simple physical description of superionic solids. Due to current limitations in diamond anvil cell techniques, the temperatures and pressures that can be investigated experimentally are too low to probe the role of hydrogen bonding in previously studied hydrides(i. e., $\mathrm{H}_{2} \mathrm{O}$ and $\mathrm{NH}_{3}$ ). On the other hands, current shock compression experiments 
have difficulty resolving transient chemical species.

Thus, our motivation to explore the high temperature-pressure properties of $\mathrm{H}_{2} \mathrm{O}$ and $\mathrm{HF}$ derives from the need to find a hydrogen bonding system that will achieve a superionic state at more accessible temperatures and pressures. Ultimately, we wish to create a simple physical picture of the rules governing this unique state of matter. HF serves as a model system for such purposes.

Although HF clusters have been studied extensively (e. g., [8]), relatively little is known about the condensed phase. In particular, few high pressure studies of HF exist, despite the relative simplicity of the molecule. A previous Raman study of solid $\mathrm{HF}$ obtained spectra at $25 \mathrm{~K}$ and up to $17 \mathrm{GPa}$ [9]. The $\mathrm{F}-\mathrm{H}$ stretch showed red-shifting and eventual disappearance as a function of pressure, concomitant with blue-shifting and broadening of the librational modes. These features were attributed to the symmetrization of the hydrogen bond, as observed in other hydrides at high pressure, including $\mathrm{DCl}[10]$, $\mathrm{HBr}[11]$ and $\mathrm{H}_{2} \mathrm{O}[6]$. This observation suggests the existence of a superionic phase of HF at previously uninvestigated pressures and temperatures.

\section{SIMULATIONS OF $\mathrm{H}_{2} \mathrm{O}$}

The density profiles of large planets, such as Uranus and Neptune, suggest that there exists within a thick layer of "hot ice", which is thought to be $56 \% \mathrm{H}_{2} \mathrm{O}$, $36 \% \mathrm{CH}_{4}$, and $8 \% \mathrm{NH}_{3}$ [12]. This has lead to theoretical investigations of the water phase diagram[2], in which Car-Parrinello Molecular Dynamics (CPMD) simulations[13] were conducted at temperature and pressures ranging from 300 to $7000 \mathrm{~K}$ and $30-300$ $\mathrm{GPa}$ [3]. At temperatures above $2000 \mathrm{~K}$ and pressures above $30 \mathrm{GPa}$, there was observed a superionic phase in which the oxygen atoms had formed a bcc lattice, and the hydrogen atoms diffused extremely rapidly (ca. $10^{-4} \mathrm{~cm}^{2} / \mathrm{s}$ ) via a hopping mechanism between oxygen lattice sites. Experimental results for the ionic conductivity of water at similar state conditions $[14,15]$ agree well with the results from ref.[3], confirming the idea of a superionic phase, and indicating a complete atomic ionization of water molecules under extreme conditions ( $\mathrm{P}>75 \mathrm{GPa}, \mathrm{T}>4000 \mathrm{~K})[15]$.

More recent $a b$ initio MD simulations were performed at temperatures up to $2000 \mathrm{~K}$ and pressures up to $30 \mathrm{GPa}[16,17]$. Under these conditions the authors found that the molecular ions $\mathrm{H}_{3} \mathrm{O}^{+}$and $\mathrm{OH}^{-}$are the major charge carriers in a fluid phase, in contrast to the bcc crystal predicted for the superionic phase. The fluid high pressure phase has been recently confirmed by X-ray diffraction results of water melting at ca. $1000 \mathrm{~K}$ and up to $40 \mathrm{GPa}$ pressure[18, 19, 20]. In addition, extrapolations of the proton diffusion constant of ice into the superionic region were found to be far lower than a superionic criteria of $10^{-4} \mathrm{~cm}^{2} / \mathrm{s}$.[21] Thus, it is clear there is great need for further work to resolve the apparently conflicting data.

We have investigated the superionic phase with more extensive first principles Car-Parrinello molecular dynamics simulations[7]. Calculated power spectra (i. e., the vibrational density of states, or VDOS) have recently been compared to measured experimental Raman spectra[22] at pressures up to $55 \mathrm{GPa}$ and temperatures of $1500 \mathrm{~K}$. The agreement between theory and experiment was very good. In particular, weakening and broadening of the $\mathrm{OH}$ stretch mode at $55 \mathrm{GPa}$ was found both theoretically and experimentally.

For our simulations, we used the CPMD code v.3.91, with the BLYP exchange-correlation functional[23, 24], and Troullier-Martins pseudopotentials[25] for both oxygen and hydrogen. A planewave cutoff of 120 Ry was employed to insure convergence of the pressure, although all other properties were seen to converge with a much lower cutoff ( $85 \mathrm{Ry}$ ). The system size was $54 \mathrm{H}_{2} \mathrm{O}$ molecules. The temperature was controlled by using Nosé-Hoover thermostats[26] for all nuclear degrees of freedom. The importance of an appropriate choice of electron mass in Car-Parrinello molecular dynamics of water has been pointed out in recent studies[27, 28]. We chose a conservative value of $200 \mathrm{au}$, and a time step of $0.048 \mathrm{fs}$. Initial conditions were generated in two ways: 1) a liquid configuration at $2000 \mathrm{~K}$ was compressed from $1.0 \mathrm{~g} / \mathrm{cc}$ to the desired density in sequential steps of $0.2 \mathrm{~g} / \mathrm{cc}$ from an equilibrated sample. 2) An ice VII configuration was relaxed at the density of interest, then heated to $2000 \mathrm{~K}$ in steps of 300 degrees each for a duration of $0.5-1$ ps. While heating the temperature was controlled via velocity scaling. We will refer to the first set of simulations as the "L" set, and the second as the "S" set. Unless stated otherwise, the results (including the pressures) from the " $\mathrm{S}$ " 
initial configurations are those reported. Once the desired density and/or temperature were achieved, all simulations were equilibrated for a minimum of 2 ps. Data collection simulations were run from 5-10 ps.

The calculated diffusion constants of hydrogen and oxygen atoms are shown in Figure 1, and the inset plot shows the equation of state for this isotherm for both "L" and "S" simulations. The two results are virtually identical up until $2.6 \mathrm{~g} / \mathrm{cc}$. At $34 \mathrm{GPa}$ $(2.0 \mathrm{~g} / \mathrm{cc})$, the hydrogen atom diffusion constant has achieved values associated with superionic conductivity (greater than $10^{-4} \mathrm{~cm}^{2} / \mathrm{s}$ ). The diffusion constant remains relatively constant with increasing density, in qualitative agreement with the experimental results of Chau et al.[15] for the ionic conductivity.

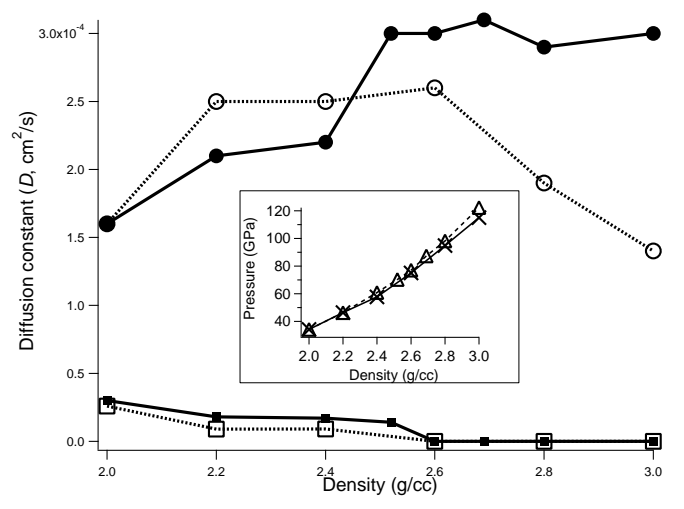

FIGURE 1. Diffusion constants for $\mathrm{O}$ and $\mathrm{H}$ atoms at $2000 \mathrm{~K}$ as a function of density. The lines with circles correspond to hydrogen, and the lines with squares to oxygen. The solid lines correspond to a liquid ('L") initial confi guration, and the dashed lines to an ice VII ('S") initial confi guration. The inset plot shows the pressure as a function of density at $2000 \mathrm{~K}$, where the triangles correspond to 'L" and the X's to 'S".

On the other hand, the $\mathrm{O}$ diffusion constant drops to zero at $75 \mathrm{GPa}(2.6 \mathrm{~g} / \mathrm{cc})$ for both " $\mathrm{L}$ " and "S" initial configurations. The surprisingly small hysteresis in the fluid to superionic transition allows us to place the transition point between $70 \mathrm{GPa}(2.5 \mathrm{~g} / \mathrm{cc})$ and 75 $\mathrm{GPa}(2.6 \mathrm{~g} / \mathrm{cc})$. The small hysteresis is most likely due to the weak O-H bonds at the conditions studied, which have free energy barriers to dissociation comparable to $k_{B} T$ (see below). Simulations which start from the "L" initial configurations are found to quench to an amorphous solid upon compression to $2.6 \mathrm{~g} / \mathrm{cc}$.
Our transition pressure of $75 \mathrm{GPa}$ is much higher than the value of $30 \mathrm{GPa}$ predicted earlier[3]. This is likely due to their use of a much smaller basis set (70 Ry). Our results are in disagreement with simple extrapolations of the proton diffusion constant to high temperatures[21].

Radial distribution functions (RDFs) for the "S" simulations are shown in Figure 2. Analysis of the oxygen-oxygen RDF for all pressures yields a coordination number of the first peak of just over 14, consistent with a high density bcc lattice in which the first two peaks are broadened due to thermal fluctuations. The RDF was further analyzed by calculating an "average position" RDF in which the position of each oxygen was averaged over the course of the trajectory. The results for $75-115 \mathrm{GPa}$ indicate the presence of bcc lattice undergoing large amplitude vibrations, even though the RDF's in Figure 2 have width similar to that of a liquid or a glass. The RDFs for the amorphous phase (not shown) are similar to those of the solid phase obtained in the "S" simulations.

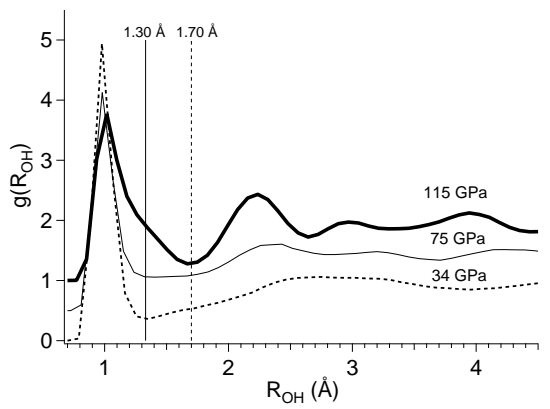

FIGURE 2. O-H radial distribution function as a function of density. At $34 \mathrm{GPa}$ we fi nd a flid state. At $75 \mathrm{GPa}$ we show a "covalent" solid phase. At $115 \mathrm{GPa}$, we fi nd a "network" phase with symmetric hydrogen bonding.

The $\mathrm{O}-\mathrm{O}$ and $\mathrm{H}-\mathrm{H}$ RDFs (not shown) indicate that no $\mathrm{O}-\mathrm{O}$ or $\mathrm{H}-\mathrm{H}$ covalent bonds are formed during the simulations at all densities. The $g\left(R_{O H}\right)$ shows a lattice-like structure at $115 \mathrm{GPa}$, which is consistent with proton diffusion via a hopping mechanism between lattice sites[3]. At $34 \mathrm{GPa}$, the coordination number for the first peak in $g\left(R_{O H}\right)$ is 2, indicating molecular $\mathrm{H}_{2} \mathrm{O}$. At $95-115 \mathrm{GPa}$, however, the coordination number for the first peak in $g\left(R_{O H}\right)$ becomes 4 , indicating that water has formed symmetric hydrogen bonds where each oxygen has four nearest neighbor hydrogens. 
Concomitant with this is a shift of the first minimum of the $\mathrm{O}-\mathrm{H}$ RDF from $1.30 \AA$ at $34 \mathrm{GPa}$ to $1.70 \AA$ at $115 \mathrm{GPa}$. We observe a similar structural change in the $\mathrm{H}-\mathrm{H}$ RDF in which the first peak lengthens from $1.63 \AA$ (close to the result for ambient conditions) to $1.85 \AA$. These observations bear a strong resemblance to the ice VII to ice $\mathrm{X}$ transition in which the covalent $\mathrm{O}-\mathrm{H}$ bond distance of ice becomes equivalent to the hydrogen bond distance as pressure is increased[29]. However, the superionic phase differs from ice $\mathrm{X}$, in that the position of the first peak in $g\left(R_{O H}\right)$ is not half the distance of the first O-O peak[29]. We analyze the effect of the change in $g\left(R_{O H}\right)$ below in terms of the molecular speciation in the simulations.

We have determined the free energy barrier for dissociation by defining a free energy surface for the oxygen-hydrogen distances, viz. $W(r)=-k_{B} T \ln \left[g\left(R_{O H}\right)\right]$, where $W(r)$ is the free energy surface (potential of mean force). The results are shown in Figure 3. The free energy barrier can then be defined as the difference in height between the first minimum and second maximum in the free energy surface. The remainder of the results discussed below are for the " $\mathrm{S}$ " simulations.

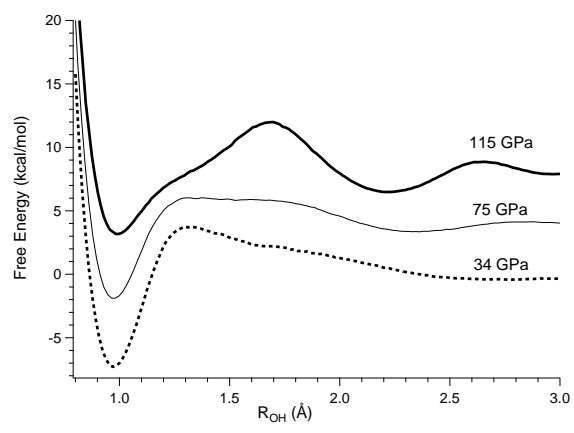

FIGURE 3. $R_{O H}$ free energy surface. The lines are spaced by a factor of $4 \mathrm{kcal} / \mathrm{mol}$ for clarity.

We now analyze the chemical species prevalent in water at these conditions. We define instantaneous species based on the $\mathrm{O}-\mathrm{H}$ bond distance. If the bond distance is less than a value $r_{c}$, we count the atom pair as bonded. Determining all the bonds in the system gives the chemical species at each point in time. Species with lifetimes less than an $\mathrm{O}-\mathrm{H}$ bond vibrational period (10 fs) are "transient", and do not represent bound molecules. The optimal cutoff $r_{c}$ between bonded and non-bonded species is given by the location of the maximum in the free energy surface[30].

The use of the free energy maximum to define a bond cutoff provides a clear picture of qualitative trends. As expected from the $g\left(R_{O H}\right)$, at $34 \mathrm{GPa}$, the free energy peak is found at $1.30 \AA$, which is approximately the same value obtained from simulations of ambient water. At $75 \mathrm{GPa}$, the free energy peak maintains almost the same position, but broadens considerably. At $115 \mathrm{GPa}$, the peak has sharpened once again, and the maximum is now at $1.70 \AA$.

Given the above definition of a bond distance, we have analyzed species lifetimes. Above $2.6 \mathrm{~g} / \mathrm{cc}$, the lifetime of all species is less than $12 \mathrm{fs}$, which is roughly the period of an $\mathrm{O}-\mathrm{H}$ bond vibration (ca. $10 \mathrm{fs}$ ). Hence, water above $75 \mathrm{GPa}$ and at $2000 \mathrm{~K}$ does not contain any molecular states, but instead forms a collection of short-lived "transient" states. The "L" simulations at $2.6 \mathrm{~g} / \mathrm{cc}$ (77 GPa) and $2000 \mathrm{~K}$ yield lifetimes nearly identical to that found in the "S" simulations described above (within $0.5 \mathrm{fs}$ ). This indicates that the amorphous states formed from the "L" simulations are closely related to the superionic bcc crystal states found in the " $\mathrm{S}$ " simulations.

Species concentrations are shown in Figure 4. At $34 \mathrm{GPa}(2.0 \mathrm{~g} / \mathrm{cc}), \mathrm{H}_{2} \mathrm{O}$ is the predominant species, with $\mathrm{H}_{3} \mathrm{O}^{+}$and $\mathrm{OH}^{-}$having mole fractions of ca. $5 \%$. In addition, some aggregation has occurred in which neutral and ionic clusters containing up to six oxygens have formed. The concentrations of $\mathrm{OH}^{-}$ and $\mathrm{H}_{3} \mathrm{O}^{+}$is low for all densities investigated, and non-existent at 95 and $115 \mathrm{GPa}(2.8$ and $3.0 \mathrm{~g} / \mathrm{cc})$. The calculated lifetimes for these species is well below 10 fs for the same thermodynamic conditions (less than 8 fs at $34 \mathrm{GPa}$ ). At pressures of 95 and 115 $\mathrm{GPa}$, the increase in the $\mathrm{O}-\mathrm{H}$ bond distance leads to the formation of extensive bond networks (Figure 5). These networks consist entirely of $\mathrm{O}-\mathrm{H}$ bonds, while $\mathrm{O}-\mathrm{O}$ and $\mathrm{H}-\mathrm{H}$ bonds were not found to be present at any point.

Maximally localized Wannier centers[31] of several trajectories were calculated, and a distribution function was determined. The outer peak at $0.46-$ $0.50 \AA$ corresponds to electrons participating in a covalent bond. Based on the above distribution, one can define the minimum at roughly $0.42 \AA$ as a dividing surface wherein a maximally localized Wannier center located at distances shorter than this, relative to its parent oxygen atom, represents a lone pair, and those found at greater distances represent cova- 


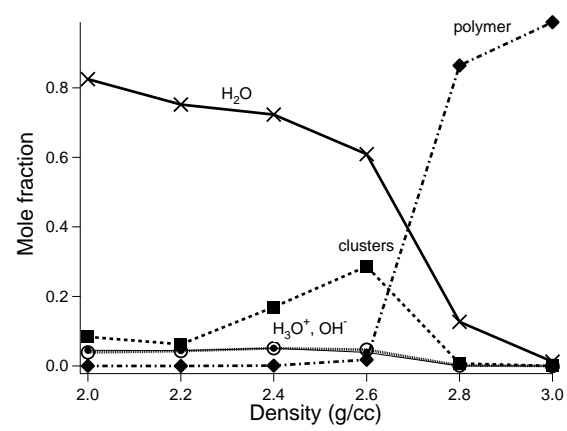

FIGURE 4. Mole fraction of species found at $34-115$ $\mathrm{GPa}$. The fi lled circles correspond to $\mathrm{H}_{3} \mathrm{O}^{+}$, while the open circles to $\mathrm{OH}^{-}$.
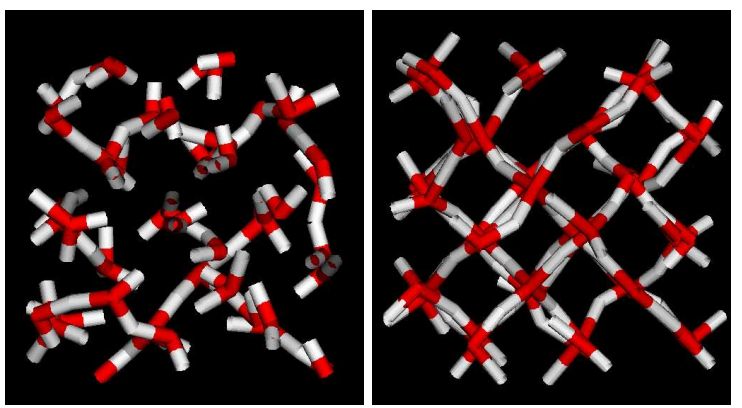

FIGURE 5. Snapshots of the simulations at $75 \mathrm{GPa}$ and $115 \mathrm{GPa}$. At $75 \mathrm{GPa}$, the water molecules are starting to cluster, and at $115 \mathrm{GPa}$, a well defi ned network has been formed. The protons dissociate very rapidly and form new clusters (at $75 \mathrm{GPa}$ ) or networks of bonds (at $115 \mathrm{GPa}$ ).

lent bonds[16]. We used this definition to compute the percentage of $\mathrm{O}-\mathrm{H}$ bonds with a Wannier center along the bond axis. Surprisingly, the results for pressures of $34-75 \mathrm{GPa}$ consistently showed that $85-95 \%$ of the $\mathrm{O}-\mathrm{H}$ bonds are covalent. For 95 and $115 \mathrm{GPa}$, we find about $50-55 \%$ of the bonds are covalent. This is consistent with symmetric hydrogen bonding, for which the split between ionic and covalent bonds would be 50/50.

\section{SIMULATIONS OF HF}

For our simulations of HF we have again used Car-Parinello Molecular Dynamics[13]. We used the BLYP exchange-correlation functional[23, 24], and Troullier-Martins pseudo-potentials[25] for both flu- orine and hydrogen. In order insure convergence of the stress tensor, we used a plane wave cutoff of 180 Rydberg. The temperature was controlled by using Nosé-Hoover thermostats[32, 26] for all nuclear degrees of freedom. A fictitious electron mass of 200 au was used. This resulted in a time step of $0.048 \mathrm{fs}$. Initial conditions were generated in two ways: 1) a configuration of $64 \mathrm{HF}$ molecules in the experimental orthorhombic crystal structure was compressed to the density of interest, and the hydrogen positions were optimized, and 2) a bcc unit lattice of two fluorine atoms was generated at the density of interest, and the hydrogen positions were similarly optimized. This bcc unit cell was then replicated so the simulation cell contained 54 molecules, and the hydrogen positions were optimized once more. The bcc lattice was employed because the orthorhombic lattice was not stable at all pressures simulated. We refer to the first set of simulation as the " $O$ " set, and the second set as the " $B$ " set. Both sets of simulations were heated to $900 \mathrm{~K}$ in steps of 300-450 K, using velocity scaling for ca. $2 \mathrm{ps}$ at each temperature step. All simulations were equilibrated for a minimum of $2 \mathrm{ps}$ at the final temperature. Data collection simulations were run for 5-10 ps.

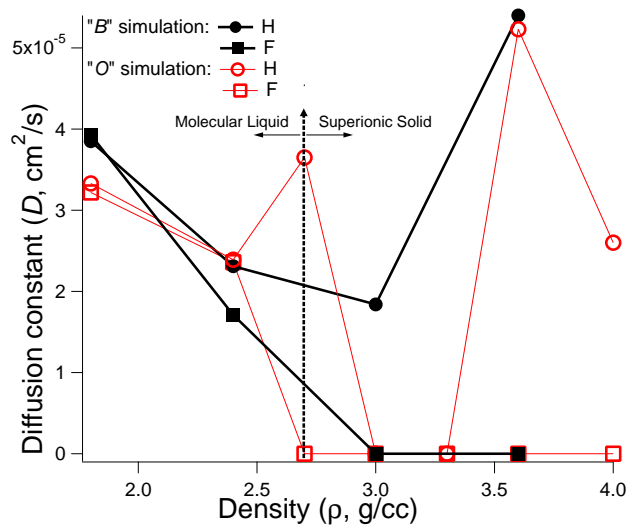

FIGURE 6. Hydrogen and florine diffusion as a function of density.

The calculated atomic diffusion constants for " $O$ " and " $B$ " simulations are shown are shown in Fig. 6 , with the equation of state results included as the inset plot of Fig. 7. The " $O$ " simulations exhibited the onset of a superionic phase at $23 \mathrm{GPa}(2.7$ $\mathrm{g} / \mathrm{cc}$ ), where the hydrogens diffuse rapidly over a disordered, "glassy" fluorine sub-lattice. At $33 \mathrm{GPa}$ $(3.0 \mathrm{~g} / \mathrm{cc})$ and $50 \mathrm{GPa}(3.3 \mathrm{~g} / \mathrm{cc})$, the simulations 
exhibited a symmetrically hydrogen bonded, "nondiffusive" state wherein the solid was no longer superionic but instead the hydrogens would vibrate along the nearest neighbor bond axis in the disordered fluorine sub-lattice. Simulations of the "nondiffusive" states were extended to $10 \mathrm{ps}$ without observing the emergence of hydrogen superionic diffusion. The lack of observable bond dissociation could be due to pressure induced increases in the activation energy for dissociation [33, 34]. At $66 \mathrm{GPa}(3.6 \mathrm{~g} / \mathrm{cc})$ and $100 \mathrm{GPa}(4.0 \mathrm{~g} / \mathrm{cc})$, the superionic hydrogen diffusion was again detected over a "glassy" fluorine state.

In comparison, at 5 and $15 \mathrm{GPa}(1.8$ and 2.4 $\mathrm{g} / \mathrm{cc}$ ), the " $B$ " simulations yield very similar diffusion constants to the " $O$ " simulations at the same pressures. However, at $33 \mathrm{GPa}$ and $66 \mathrm{GPa}$, we observe in the " $B$ " simulations a stable fluorine bcc lattice. For both pressures, the hydrogens exhibit diffusion comparable with molecular diffusion at ambient conditions[35]. Interestingly, the " $O$ " and " $B$ " simulations at each density investigated, including "non-diffusive" vs. superionic states, yielded average potential energies within $1 \mathrm{kcal} / \mathrm{mol}$ of each other. The superionic state is likely entropically favored at these conditions (we estimate $T \Delta S \approx 2 \mathrm{kcal} / \mathrm{mol}$ ). The bcc lattice appears to have high energetic barriers to melting. We found that in order to melt the " $B$ " system, at $3.0 \mathrm{~g} / \mathrm{cc}$ the simulation had to be heated via Nosé-Hoover thermostats to $1900 \mathrm{~K}$ (in steps of 500 degrees for $2 \mathrm{ps}$ ) before melting was observed. This simulation was then similarly cooled to $600 \mathrm{~K}$ in steps of $100-200$ degrees for 2-4 ps, at which point the fluorines form an amorphous solid. This places the actual superionic phase transition at $33 \mathrm{GPa}$ between $600-900 \mathrm{~K}$.

The vibrational density of states (VDOS) for two of the " $B$ " simulations are presented in Figure 7. As pressure is increased, the spectra show that HF is forming a symmetrically hydrogen bonded solid. At $5 \mathrm{GPa}$, the first peak in the VDOS occurs at ca. $125 \mathrm{~cm}^{-1}$ with a noticeable shoulder at ca. 500 $\mathrm{cm}^{-1}$, both of which correspond closely to the experimental Raman spectrum translational and librational bands at the same pressure[9]. In addition, we observe the H-F stretch vibrational band at ca. $3400 \mathrm{~cm}^{-1}$, which matches well with the both the experimental IR [36] spectra and Raman spectra. In contrast, at $66 \mathrm{GPa}$ dramatic changes have occurred within the system. The translational band is absent,

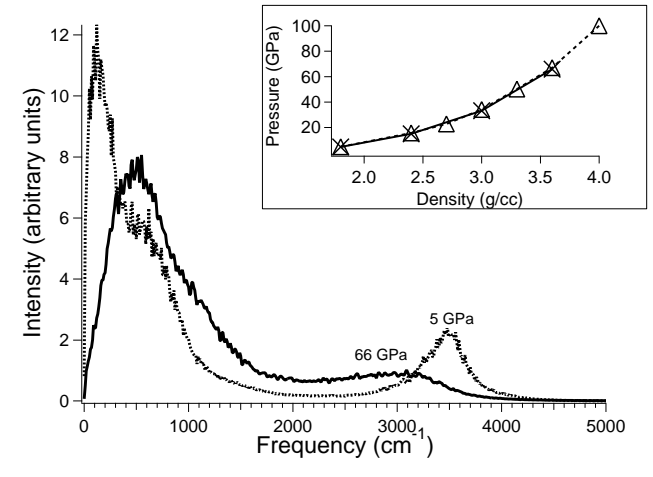

FIGURE 7. Vibrational density of states of " $B$ " simulations at $5 \mathrm{GPa}$ and $66 \mathrm{GPa}$. In the equation of state inset plot, the X's correspond to the " $B$ " simulations and the open triangles to the " $\mathrm{O}$ " simulations.

and in place of the librational "shoulder" there is a broad band at $600 \mathrm{~cm}^{-1}$. In addition, the $\mathrm{H}-\mathrm{F}$ stretch peak is red-shifted and significantly decreased in intensity, a feature also found in the high pressure Raman studies[9]. As stated above, these two symmetric H-bond features have been observed in low temperature, high pressure experiments on various hydrogen bonded systems $[9,10,11,6]$.

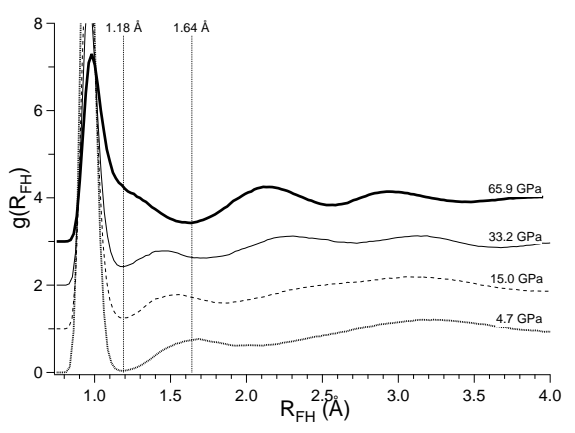

FIGURE 8. F-H Radial distribution functions for the "B" simulations.

The $\mathrm{F}-\mathrm{H}$ radial distribution functions (RDF) for the " $B$ " simulations are shown in Figure 8. The $g\left(R_{F H}\right)$ exhibits a lattice-like structure, wherein the hydrogens are hopping between specific sites relative to the fluorines. At $5 \mathrm{GPa}$, the first minimum of $g\left(R_{F H}\right)$ is at ca. $1.2 \AA$, with a coordination number of 1 , indicating the predominance of molecular HF. At $66 \mathrm{GPa}$, the first minimum has shifted to $1.64 \AA$, and the coordination number to 2 , consistent with hydrogen bond symmetrization. The first peak at $66 \mathrm{GPa}$ 
can be decomposed into two Gaussians, one centered at ca. $1 \AA$, and the other at ca. $1.2 \AA$. The first Gaussian most likely corresponds to a covalent bond, and the peak at $1.2 \AA$ to an ionic bond with the neighboring fluorine. Thus, the hydrogen bonding in superionic HF is not entirely symmetrized in that the covalent and ionic bond lengths are not yet equal.

We define instantaneous species based on the F$\mathrm{H}$ bond distance. If the bond distance is less than a value $r_{c}$, we count the atom pair as bonded. Determining all the bonds in the system gives the chemical species at each point in time. Considering the $\mathrm{F}-\mathrm{H}$ vibrational frequency at $3400 \mathrm{~cm}^{-1}$, species with lifetimes less than an $\mathrm{F}-\mathrm{H}$ bond vibrational period (10 fs) are "transient", and do not represent bound molecules. The optimal cutoff $r_{c}$ between bonded and non-bonded species is given by the location of the maximum in the free energy surface, viz. $W(r)=-k_{B} T \ln \left[g\left(R_{F H}\right)\right]$, where $W(r)$ is the free energy surface (potential of mean force). The maximum in $W(r)$ corresponds to the first minimum in $g\left(R_{F H}\right)$ [7]. This choice, while intuitive, also corresponds to the optimal choice of a transition state for the dissociation[30] within transition state theory.

Our result for $3.6 \mathrm{~g} / \mathrm{cc}$ is the most interesting state point investigated. At this density, the predominant species is a transient network of F-H bonds (Fig. 9), bearing striking resemblance to our recent results for superionic water[7]. This network is due to the increase in $\mathrm{F}-\mathrm{H}$ bond distance; no $\mathrm{F}-\mathrm{F}$ or $\mathrm{H}-\mathrm{H}$ bonds were found at any point during the simulation. Although significant quantities of HF were observed, its lifetime was calculated to be ca. 7 fs. Significant clusters were again observed, although the predominant species by far were HF and the network solid. We find that at $3.6 \mathrm{~g} / \mathrm{cc}$, hydrogens diffuse via a trigonal coordination mechanism. This is consistent with features of well-known superionic solids of similar ionic character, such as AgI, in which the silver ion diffuses through the trigonal interstitial sites[1]. Snapshots of a hydrogen diffusing through the lattice are shown in Fig. 9.

Finally, we have performed a Mulliken[37] population analysis of a snapshot of the " $B$ " simulation at $3.6 \mathrm{~g} / \mathrm{cc}$ in order to estimate the ionic charges[38, 39]. For a single snapshot, we observe an average hydrogen charge (in au) of $+0.43 \pm 0.033$ and an average fluorine charge of $-0.43 \pm 0.049$. Again, results for the " $O$ " simulations were nearly identical. Interestingly, we observed that a geometry optimized HF
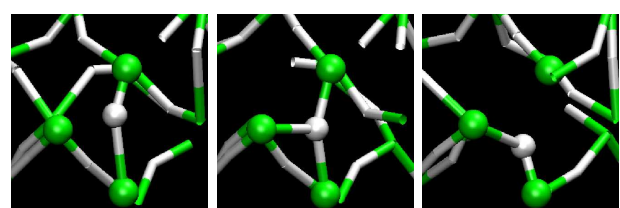

FIGURE 9. Sequential snapshots of superionic HF, taken from the "B" simulations at $66 \mathrm{GPa}(3.6 \mathrm{~g} / \mathrm{cc})$. Fluorines are labeled green and the hydrogens are white. In (b), a triply coordinated hydrogen is found. This transition state confi guration has a lifetime of only ca. $10 \mathrm{fs}$. Part (c) shows the new $\mathrm{H}-\mathrm{F}-\mathrm{H}$ bond pair.

monomer has nearly identical charges on the atomic species. We expect that the site charges will approach an absolute value of 0.5 as the hydrogen bonding becomes increasingly symmetric. Similar studies for $\mathrm{H}_{2} \mathrm{O}$ and other hydrides are currently underway.

\section{CONCLUSIONS}

In conclusion, we have performed first principles simulations of water at pressures up to $115 \mathrm{GPa}$ (3.0 $\mathrm{g} / \mathrm{cc}$ ) and $2000 \mathrm{~K}$. Along this isotherm we can define three different phases. First, from $34 \mathrm{GPa}$ to $58 \mathrm{GPa}$ $(2.0-2.4 \mathrm{~g} / \mathrm{cc})$, we observe a molecular fluid phase with superionic diffusion of the hydrogens. Second, at $75 \mathrm{GPa}(2.6 \mathrm{~g} / \mathrm{cc})$, we find a stable bcc oxygen lattice with superionic proton conduction. $\mathrm{O}-\mathrm{H}$ bonds within this "solid" phase are found to be mostly covalent, despite their exceedingly short lifetimes of ca. $10 \mathrm{fs}$. Third, at $95-115 \mathrm{GPa}(2.8-3.0 \mathrm{~g} / \mathrm{cc})$ we find a transformation to a phase dominated by transient networks of symmetric O-H hydrogen bonds. Given the smooth nature of the calculated P-V isotherm, the transition to the network phase does not appear to be first order. The network can be attributed to the symmetrization of the hydrogen bond, similar to the ice VII to ice $\mathrm{X}$ transition

We have also discovered a new superionic solid form of HF, wherein we observe a stable fluorine bcc lattice, and a highly mobile hydrogen phase. At the highest pressures simulated, we see a symmetrization of the hydrogen bond, where the superionic solid becomes an extended network of very short-lived partially covalent bonds. The hydrogens diffuse through the fluorine sub-lattice via the trigonal interstitial sites. Superionic HF should be ob- 
servable by experiments at ca. $23 \mathrm{GPa}$ and $900 \mathrm{~K}$ (c. f. $49 \mathrm{GPa}$ and $1000 \mathrm{~K}$ for water[6]). The symmetrically hydrogen bonded form of the solid is predicted to occur at $66 \mathrm{GPa}$ (c. f. $95 \mathrm{GPa}$ for water[7]). In addition, the hydrogens and fluorines are not fully ionized, similar to previous results for a superionic solid[40].

The temperatures and pressures needed to induce superionic diffusion in HF are significantly lower than what is required for other known superionic hydrides[3, 6]. Through superionic HF, we have discovered a model system wherein significant changes in the hydrogen bonding occur at experimentally achievable conditions. Our and future studies could have profound effects on our understanding of the nature of superionic conduction by allowing us to identify simple rules that govern the properties of rapid solid state diffusion.

This work was performed under the auspices of the U. S. Department of Energy by the University of California Lawrence Livermore National Laboratory under contract No. W-7405-Eng-48.

\section{REFERENCES}

1. S. Hull, Rep. Prog. Phys. 67, 1233 (2004).

2. P. Demontis, R. LeSar, and M. L. Klein, Phys. Rev. Lett. 60, 2284 (1988).

3. C. Cavazzoni, G. L. Chiarotti, S. Scandolo, E. Tosatti, M. Bernasconi, and M. Parrinello, Science 283, 44 (1999).

4. B. Schwager, L. Chudinovbskikh, and R. Boehler, J. Phys.: Condens. Matter 16, 1177 (2004).

5. J.-F. Lin, E. Gregoryanz, V. V. Struzhkin, M. Somayazulu, H. k. Mao, and R. J. Hemley, Geophys. Res. Lett. 32, 11306 (2005).

6. A. F. Goncharov, N. Goldman, L. E. Fried, J. C. Crowhurst, I.-F. W. Kuo, C. J. Mundy, and J. M. Zaug, Phys. Rev. Lett. 94, 125508 (2005).

7. N. Goldman, L. E. Fried, I.-F. W. Kuo, and C. J. Mundy, Phys. Rev. Lett. 94, 217801 (2005).

8. J. R. Dyke, B. J. Howard, and W. Klemperer, J. Chem. Phys. 56, 2442 (1972).

9. D. A. Pinnick, A. I. Katz, and R. C. Hanson, Phys. Rev. B 39, 8677 (1989).

10. E. Katoh, H. Yamawaki, H. Fujihisa, M. Sakashita, and K. Aoki, Phys. Rev. B 61, 119 (2000).

11. T. Ikeda, M. Sprik, K. Terakura, and M. Parrinello, J. Chem. Phys. 111, 1595 (1999).

12. W. B. Hubbard, Science 214, 145 (1981).

13. R. Car and M. Parrinello, Phys. Rev. Lett. 55, 2471 (1985).
14. W. J. Nellis, N. C. Holmes, A. C. Mitchell, D. C. Hamilton, and M. Nicol, J. Chem. Phys 107, 9096 (1997).

15. R. Chau, A. C. Mitchell, R. W. Minich, and W. J. Nellis, J. Chem. Phys 114, 1361 (2001).

16. E. Schwegler, G. Galli, F. Gygi, and R. Q. Hood, Phys. Rev. Lett. 87, 265501 (2001).

17. C. Dellago, P. L. Geissler, D. Chandler, J. Hutter, and M. Parrinello, Phys. Rev. Lett. 89, 199601 (2001).

18. B. Schwager, L. Chudinovskikh, A. Gavriliuk, and R. Boehler, J. Phys: Condensed Matter 16, S1177 (2004).

19. M. Frank, Y. Fei, and J. Z. Hu, Geochemica et Cosmochimica Acta 68, 2781 (2004), submitted.

20. M. R. Frank, B. Militzer, Y. Fei, J. Hu, and R. Hemley, Phys. Rev. Lett. (2004), submitted.

21. E. Katoh, H. Yamawaki, H. Fujihisa, M. Sakashita, and K. Aoki, Science 295, 1264 (2004).

22. A. F. Goncharov, N. Goldman, L. E. Fried, J. C. Crowhurst, I.-F. W. Kuo, C. J. Mundy, and J. M. Zaug, Phys. Rev. Lett. (2004), submitted.

23. A. D. Becke, Phys. Rev. A 38, 3098 (1988).

24. C. Lee, W. Yang, and R. G. Parr, Phys. Rev. B 37, 785 (1988)

25. N. Troullier and J. Martins, Phys. Rev. B 43, 1993 (1991).

26. W. G. Hoover, Phys. Rev. A 31, 1695 (1985).

27. J. Grossman, E. Schwegler, E. W. Draeger, F. Gygi, and G. Galli, J. Chem. Phys. 120, 300 (2004).

28. I.-F. Kuo, C. Mundy, M. McGrath, J. Siepmann, J. VandeVondele, M. Sprik, J. Hutter, B. Chen, M. Klein, F. Mohamed, M. Krack, and M. Parrinello, J. Phys. Chem. B 108, 12990 (2004).

29. M. Benoit, A. H. Romero, and D. Marx, Phys. Rev. Lett. 89, 145501 (2002).

30. D. Chandler, J. Chem. Phys. 68, 2959 (1978).

31. P. L. Silvestrelli and M. Parrinello, Phys. Rev. Lett. 82, 3308 (1999).

32. S. Nosé, Mol. Phys. 52, 255 (1984).

33. R. A. Secco, E. A. Secco, and Q. Chen, Solid State Ionics 110, 283 (1998).

34. R. J. Hemley, Annu. Rev. Phys. Chem. 51, 763 (2000).

35. N. Karger, T. Vardag, and H.-D. Lüdemann, J. Chem. Phys. 100, 8271 (1994).

36. D. K. Buslov, N. I. Sushko, and G. V. Yukhnevich, J. Opt. Tech. 70, 28 (2003).

37. R. S. Mulliken, J. Chem. Phys. 23, 1833 (1955).

38. The mixed basis set $\mathrm{CP} 2 \mathrm{~K}$ code was used to perform the Mulliken population analysis, with a cutoff of 400 Ry, a basis set of DZVP, and with the BLYP functional. See http://www.berlios.de.

39. J. VandeVondele, M. Krack, F. Mohamed, M. Parrinello, T. Chassaing, and J. Hutter, Comp. Phys. Comm. 167, 103 (2005).

40. F. Shimojo and M. Aniya, J. Phys. Soc. Japan 72, 2702 (2003). 